\title{
SuAEDA EDUlis (ChenOPODIACEAE), UNA NUEVA ESPECIE DE LAGOS SALINOS DEL CENTRO DE MÉXICO
}

\author{
Roberto Noguez-Hernández ${ }^{1}$ Aquiles Carballo-Carballo² e Hilda Flores-Olvera 3 3,4 \\ 'Departamento de Preparatoria Agrícola. Universidad Autónoma Chapingo, Chapingo, Estado de México, México \\ ${ }^{2}$ Producción de Semillas. Colegio de Postgraduados. Campus Montecillo, Montecillo, Estado de México, México \\ ${ }^{3}$ Departamento de Botánica, Instituto de Biología. Universidad Nacional Autónoma de México, México, D.F., México \\ ${ }^{4}$ Autor para la correspondencia: mahilda@ibiologia.unam.mx
}

\begin{abstract}
Resumen: Se describe e ilustra una nueva especie de Suaeda sect. Brezia, Suaeda edulis Flores Olv. \& Noguez. La especie se distribuye en lagos salinos de Guanajuato, Jalisco, Michoacán, Estado de México, Distrito Federal, Tlaxcala y Puebla. Suaeda edulis fue confundida con S. nigra (= S. torreyana) y con S. mexicana, pero S. edulis es similar a S. calceoliformis y se caracteriza por ser una hierba anual, erecta a ascendente, con flores zigomorfas y uno a tres segmentos del perianto con alargamientos apicales como cornículos y, en fruto, con alas transversales en la base evidentemente nervados. Las flores tienen 2-3 estambres y están dispuestas en glomérulos axilares, de 1 a 5 flores, en toda la planta, algunas veces arregladas en espigas terminales y axilares; las semillas son de 0.9-1.6 mm de diámetro y el número cromosómico es de $2 n=54$. Es comestible y se recolecta de poblaciones silvestres o bajo cultivo en la zona agrícola del Distrito Federal en donde se le llama "romerito".
\end{abstract}

Palabras clave: planta comestible, Suaeda sect. Brezia, Suaeda calceoliformis, Suaeda mexicana, Suaeda nigra.

\begin{abstract}
A new species of Suaeda sect. Brezia, Suaeda edulis Flores Olv. \& Noguez is described and illustrated. It occurs in saline lakes of Guanajuato, Jalisco, Michoacán, Estado de Mexico, Distrito Federal, Tlaxcala and Puebla. Suaeda edulis was confused with $S$. nigra (= S. torreyana) and S. mexicana, but it is more similar to S. calceoliformis; it is an annual, erect to ascending herb; the flowers are zygomorphic, with one to three perianth segments that are enlarged apically in a horn-like appendices, in fruit with transverse nerved wings near the base. The flowers are clustered axillary in groups of 1-5, have 2-3 stamens, and are distributed throughout the plant, sometimes in terminal and axillar spikes; the seeds are 0.9-1.6 mm diameter and the chromosome number is $2 n=54$. It is an edible herb harvested from wild populations, but it is also cultivated in the agricultural area of the Distrito Federal where it is known as "romerito".
\end{abstract}

Key words: edible plant, Suaeda sect. Brezia, Suaeda calceoliformis, Suaeda mexicana, Suaeda nigra.

E género Suaeda Forssk. ex Scop., con alrededor de 110 especies, pertenece a la subfamilia Suaedoidae Ulbr. de la familia Chenopodiaceae (Schütze et al., 2003). Crece generalmente en zonas húmedas salinas o alcalinas y tiene una distribución cosmopolita en la costa del mar (Reed, 1979; Ferren y Schenk, 2004), así como en el interior en las partes bajas de cuencas endorreicas (Rzedowski, 1978). El género comprende hierbas anuales o perennes, subarbustos y arbustos, con tallos erguidos o postrados, glabros o pubescentes, hojas sésiles o subsésiles, alternas u opuestas, enteras, carnosas, aplanadas o semiteretes a teretes, flores inconspicuas con perianto actinomorfo o zigomorfo o irregular, de cinco segmentos, verdes, suculentos, persistentes y envolviendo al fruto, (1-2-) 5 estambres, 2-5 estigmas; flores arregladas en glomérulos en las axilas de brácteas foliares comúnmen-

te arregladas en espigas o algunas veces racimos y utrículos con pericarpo membranáceo y semillas algunas veces dimórficas (Ferren y Schenk, 2004).

Suaeda incluye especies polimórficas, cuya variación parece que es debida a factores ambientales, pero probablemente también a diferencias genéticas (Ferren y Schenk, 2004). Reconociendo la confusión taxonómica del género, Hopkins y Blackwell (1977) sugirieron que los caracteres que son constantes y presumiblemente confiables a nivel de especie parecen ser el hábito herbáceo contra sufrutescente, el tipo de inflorescencia (incluyendo la densidad de los glomérulos), la forma de la hoja y longitud de los entrenudos, características del cáliz y tamaño de la semilla. Pedrol y Castroviejo (1988) indicaron que estudios de diversos autores sobre los efectos de los factores ambientales en diferen- 
tes especies de Suaeda, mostraron que la altura y número de los tallos, suculencia, grosor, longitud y forma de las hojas, varían según la salinidad del medio. Lomonosova y Freitag (2003) también observaron que la forma y tamaño de las hojas, así como el tamaño de las plantas y de los entrenudos son afectados considerablemente en Suaeda tschujensis Lomon. \& Freitag y en otras especies anuales de Suaeda, por la salinidad, suministro de agua y nutrientes. Los caracteres del perianto, incluyendo diámetro y ornamentaciones, de las brácteas, del número de flores por glomérulo, de la disposición de las flores y del tamaño de la semilla, fueron considerados para distinguir especies de Suaeda de Norteamérica (Ferren y Schenk, 2004).

Con base en análisis moleculares, Schütze et al. (2003) propusieron una clasificación infragenérica para Suaeda, apoyada por caracteres morfológicos, reconociendo siete secciones. De acuerdo con estos autores, en Norteamérica se distribuyen siete especies de S. sect. Brezia (Moq.) Volk. in Engl. \& Prantl así como seis de S. sect. Salsina Moq. Por su parte, Ferren y Schenk (2004) en el tratamiento taxonómico para las especies de Suaeda de Norteamérica, norte de México, coinciden en el reconocimiento de $S$. sect. Brezia pero reconocen $S$. sect. Limbogermen Iljin, que fue incluida en $S$. sect. Salsina por Schütze et al. (2003). El tratamiento taxonómico de las especies de Suaeda por Ferren y Schenk (2004) significa un avance substancial para una monografía de las especies mexicanas que estamos iniciando.

Dentro del Proyecto de Tesis Doctoral por el primer autor de esta contribución y de la Red Romerito del Sistema Nacional de Recursos Fitogenéticos para la Alimentación y la Agricultura (SINAREFI), se encontró una nueva especie de Suaeda con importancia económica en el Distrito Federal y estado de México que se describe en este artículo.

Suaeda edulis Flores Olv. \& Noguez sp. nov. (Figura 1). Annual herb, erect to ascending, similar to Suaeda calceoliformis. Notably different by the glomes 1-5-flowered, smaller flowers, with 2-3 stamens, asymmetric and nerved mature perianth segments; flowers clustered in axillar glomerules distributed throughout the plant or in spikes to 3 $\mathrm{cm}$ length, seeds of 0.9-1.6 mm, and chromosome number $2 n=54$.

TIPO: México, Estado de México, Lago de Texcoco, alt. 2,350 m, pastizal halófilo de Distichlis spicata, hierba anual, erecta, suculenta, rojiza, de hasta 1.15 m, 31/08/2010. M.H. Flores-Olvera 1634 y R. Noguez Hernández (Holotipo: MEXU; Isotipos: B, CHAPA, MO, TEX, UAM-I, XAL, XOLO).

Hierba estrictamente anual de $15-110 \mathrm{~cm}$ de alto, erecta a ascendente, plantas de color verde, glauco o rojizas, tornándose pardas con la edad; glabras. Tallos no ramificados o 13 (-7) veces ramificados, en la base o a lo largo del tallo, más cortos o igualando al tallo principal; estriados con líneas de color verde, pardo o rojizo. Hojas alternas, suculentas, linea- res o angostamente lanceoladas, más anchas en la base, las que sostienen ramas floríferas de 10.0-54.0 × 0.5-2.0 mm; margen membranáceo a todo lo largo, blanquecino; ápice apiculado. Inflorescencias en glomérulos axilares de 1-3 (5) flores, a todo lo largo de las ramas o dispuestas en espigas de 1.0-3.0 cm de largo, algunas veces dando una apariencia paniculiforme por el acortamiento hacia la parte terminal de las ramas laterales; brácteas similares a las hojas superiores pero gradualmente más cortas, comúnmente, las que sostienen glomérulos angostamente lanceoladas, más anchas en la base, de 2.0-12.0 × 1.0-1.8 mm, margen membranáceo, base envolvente. Bractéolas hialinas de 0.75-1.75 × 1.0-2.5 mm, ovadas o triangulares, irregularmente eroso-dentadas con ápice agudo a caudado. Flores hermafroditas, zigomorfas, la parte más larga de 0.5-0.7 mm en antesis y de 0.8-3.0 $\mathrm{mm}$ en etapa madura. Segmentos del perianto suculentos, fusionados hasta la mitad, verde-glauco, cóncavos, 1 a 3 provistos de cornículos distales, un segmento más grande con el cornículo más largo, cubriendo los dos laterales; en fruto son evidentemente nervados con alas abaxiales transversales. Estambres 2-3, 0.27-0.44 mm, exsertos. Ovario depreso-ovoide; estigmas 2, papilosos, surgiendo del ápice atenuado del ovario. Semillas dimórficas, horizontales, negras o pardo claro, lenticulares, de 0.9-1.6 mm. $2 n=54$ (Noguez et al. datos sin publicar).

Distribución y ecología. Suaeda edulis se distribuye en lagos salinos de Guanajuato, Jalisco, Michoacán, México, Distrito Federal, Tlaxcala y Puebla. Crece en suelos inundados y húmedos en pastizales halófilos con Distichlis spicata a intervalo altitudinal de 1,347 a 2,351 m (Figuras 2A-B, 3). Se desarrolla perfectamente en cultivo (Figura 2C) en el Distrito Federal en San Andrés Mixquic, San Pedro Tláhuac, San Gregorio Atlapulco y Santiago Tulyehualco. Florece y fructifica de mayo a octubre.

Etimología. El epíteto específico de esta especie deriva de "edulis" por ser consumida, tradicionalmente, en el Distrito Federal y en el Estado de México, ya sea recolectada de poblaciones silvestres o cultivadas. También se recolecta para consumo en los alrededores del Lago Totolcingo en los estados de Tlaxcala y Puebla.

Nombres comunes y usos. En el Distrito Federal y el Estado de México, esta especie se conoce como "romerito" (Martínez, 1979; Martínez y Matuda, 1979), "romerillo", "romerito" o "romero" (Calderón de Rzedowski, 2001) y en los alrededores del Lago Totolcingo, Puebla se le nombra "manguinillo". Es una planta comestible que se utiliza especialmente en celebraciones religiosas: la Semana Santa y la Navidad. Las partes terminales de los tallos se consumen cocidas en platillos típicos de estas fechas festivas. Datos etnobotánicos sugieren que fue utilizada en la época prehispánica (Rzedowski y Equihua, 1987; Bye y Linares, 2000). 


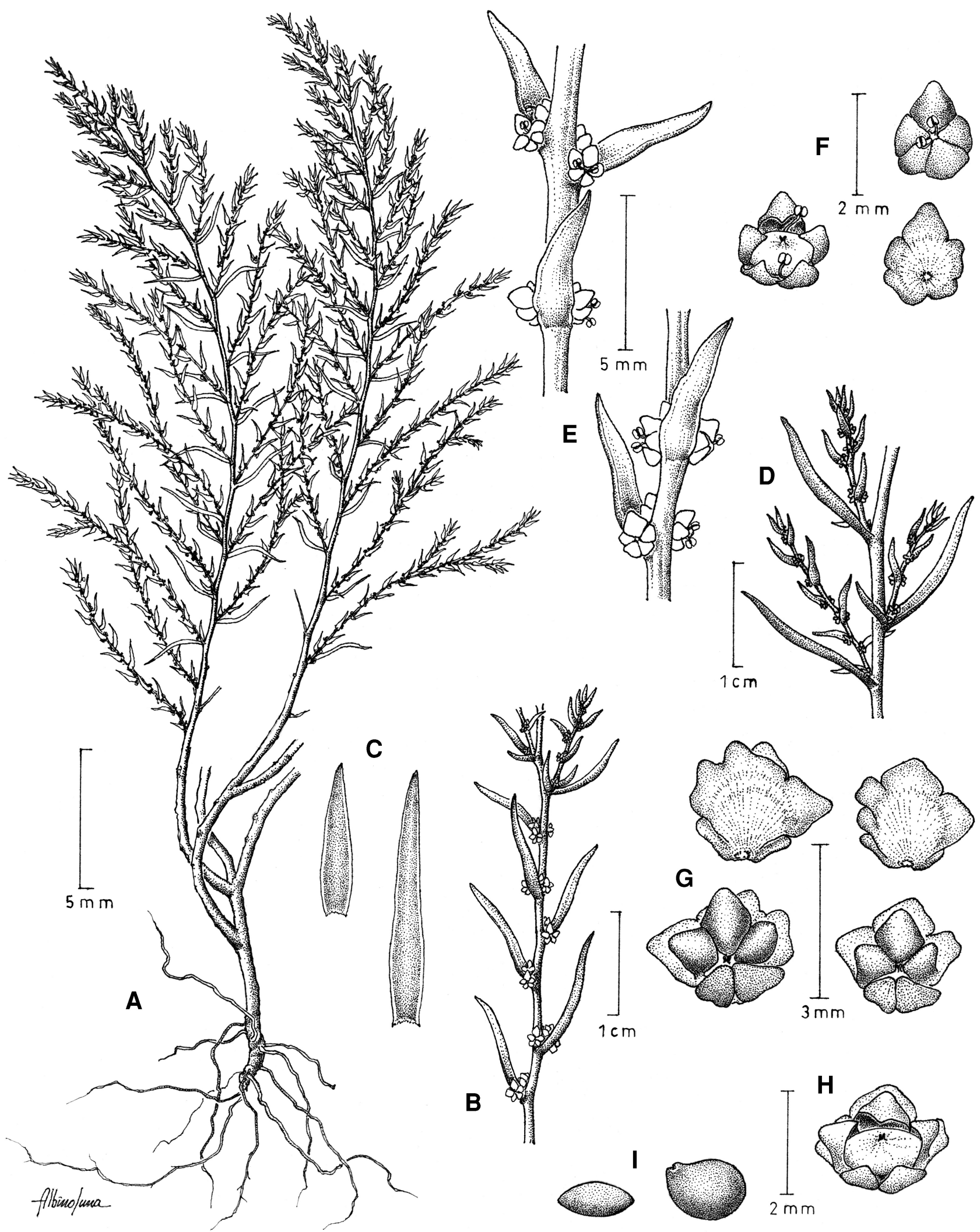

Figura 1. Suaeda edulis Flores Olv. et Noguez. A. Aspecto de la planta en floración. B. Rama fértil. C. Brácteas foliares que sostienen a los glomérulos. D. Ramas con hojas que sostienen espigas. E. Ramas con flores en antesis. F-H. Flores en distintas vistas y etapas de desarrollo. I. Semillas. 

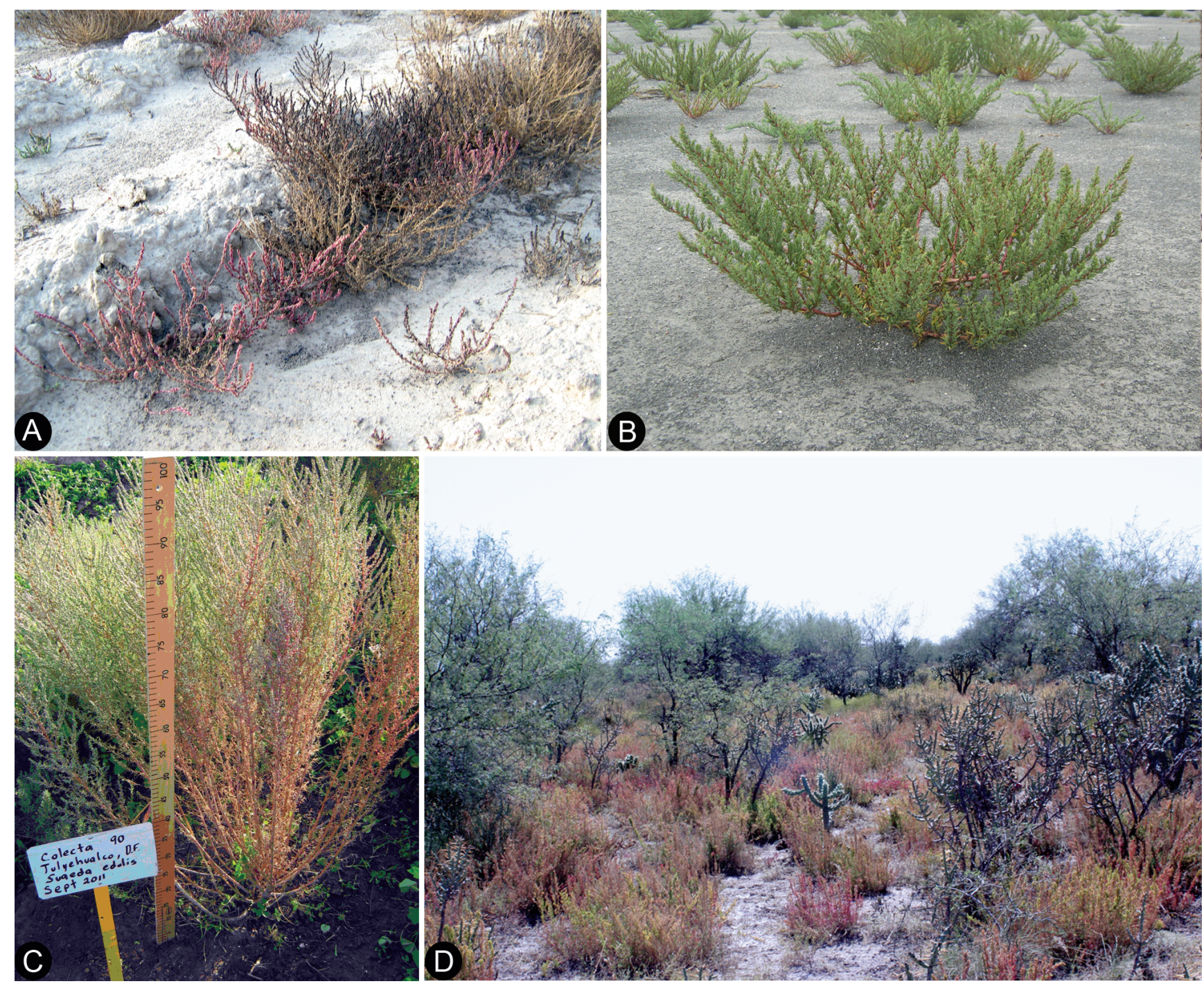

Figura 2. Plantas vivas de Suaeda. A-C. Suaeda edulis. A. En hábitat natural en Texcoco, Estado de México. B. En hábitat natural en Sayula, Jalisco. C. Bajo cultivo en Montecillo, Estado de México. D. Suaeda mexicana en terrenos de la ex Hacienda de Angostura, Río Verde, San Luis Potosí (localidad tipo).

Discusión. Las poblaciones de Suaeda del Distrito Federal y Estado de México fueron identificadas como: $S$. diffusa S.Watson (Reiche, 1977; Sánchez, 1978; Martínez, 1979; Martínez y Matuda, 1979), S. mexicana (Standl.) Standl. (Martínez, 1979; Calderón de Rzedowski, 2001) o S. torreyana S.Watson (Ramírez y Alcozer, 1902; Rzedowski, 1978; Martínez, 1979; Calderón de Rzedowski, 1979; Espinosa y Sarukhán, 1997; Hanan-Alipi et al., 2009). De estos nombres, $S$. diffusa y $S$. torreyana, han sido reducidos a sinónimos de $S$. nigra (Raf.) J.F.Macbr. (Hopkins y Blackwell, 1977; Ferren y Schenk, 2004), mientras que $S$. mexicana es reconocida para México, sin que se conozca con precisión la distribución. Tanto $S$. mexicana como $S$. nigra corresponden a diferentes secciones del género por diferir en tipo de anatomía de la hoja, hábito, simetría de la flor, caracteres de los segmentos del perianto y del estigma (Ferren y Schenk, 2004; Schütze et al., 2003).

Suaeda nigra (= S. diffusa, S. moquinii (Torr.) Greene, $S$. torreyana) (Hopkins y Blackwell, 1977; Ferren y Schenk, 2004), pertenece a $S$. sect. Salsina por tener anatomía $C_{4}$ tipo Salsina, los segmentos el perianto sin apéndices, los estigmas insertos en una depresión del ápice del ovario (Schütze et al., 2003) y por el perianto actinomorfo (Ferren y Schenk, 2004). Esta especie se distingue por la bráctea que sostiene a los glomérulos, que es más corta que las hojas proximales y por las inflorescencias en ramas distales más delgadas que las ramas vegetativas. Es considerada como arbustiva, subarbustiva o anual facultativa, tiene una gran 


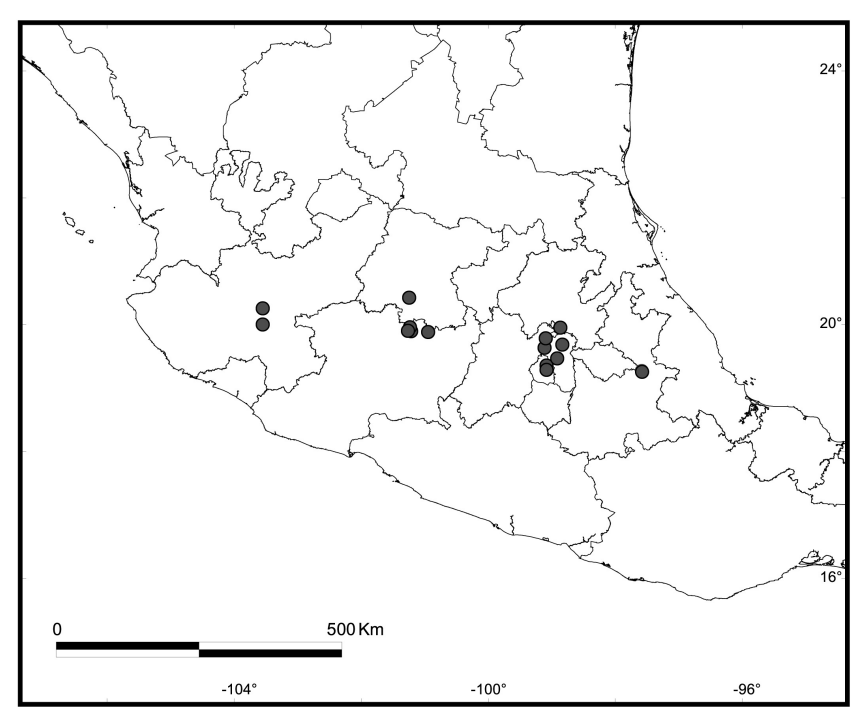

Figura 3. Distribución de Suaeda edulis. plasticidad fenotípica y está ampliamente distribuida en el este de los Estados Unidos de América y en México, lo que ha propiciado numerosos sinónimos (Hopkins y Blackwell, 1977; Ferren y Schenk, 2004).

Las poblaciones de Suaeda de Guanajuato, Jalisco, Michoacán, Estado de México, Distrito Federal, Tlaxcala y Puebla se ubican, al igual que $S$. mexicana, en $S$. sect. Brezia por tener anatomía $\mathrm{C}_{3}$ tipo Brezia, los estigmas insertos sobre el ápice atenuado del ovario (Schütze et al., 2003), el perianto zigomorfo, los tépalos redondeados abaxialmente, con prolongaciones distales como cornículos y/o alas transversales hacia la base (Ferren y Schenk, 2004).

Si bien Suaeda mexicana fue descrita como anual (Standley, 1916), tanto el holotipo depositado en el U. S. National Herbarium (US), como los isotipos en el Herbario Nacional de México (MEXU) y en Missouri Botanical Garden (MO), carecen de la base del tallo y de la raíz y solamente tienen la parte terminal de la planta. En el ejemplar de MEXU los tallos son de consistencia leñosa. Las

Cuadro 1. Caracteres que distinguen a Suaeda edulis de las especies similares y de las que se ha confundido.

\begin{tabular}{|c|c|c|c|c|c|}
\hline Caracteres & S. edulis & S. calceoliformis & S. occidentalis & S. mexicana & S. nigra \\
\hline Duración & anual & anual & anual & perenne & $\begin{array}{l}\text { perenne o } \\
\text { facultativa anual }\end{array}$ \\
\hline Hábito & erecta o ascendente & postrada o erecta & erecta o decumbente & decumbente & decumbente a erecta \\
\hline Altura de la planta $(\mathrm{cm})$ & $15-110$ & $5-100$ & $5-35$ & $30-150$ & $20-150$ \\
\hline Tipo de hoja & C3 tipo Brezia & C3 tipo Brezia & C3 tipo Brezia & C3 tipo Brezia & C4 tipo Salsina \\
\hline Posición de las hojas & alternas & alternas & alternas & $\begin{array}{l}\text { opuestas hacia } \\
\text { la base }\end{array}$ & alternas \\
\hline Largo de las hojas (mm) & $10-54$ & (5-)10-40 & $5-30$ & $12-45$ & (5-)10-30 \\
\hline $\begin{array}{l}\text { Número de flores } \\
\text { por glomérulo }\end{array}$ & $1-3(-5)$ & $3-5(-7)$ & $1-3$ & $1-5$ & $1-12$ \\
\hline $\begin{array}{l}\text { Tamaño de las } \\
\text { espigas }(\mathrm{cm})\end{array}$ & $1-3$ & $1-6$ & $1-6$ & $2-10.5$ & $1-4.5$ \\
\hline Tipo de inflorescencia & $\begin{array}{l}\text { glomérulos axilares en } \\
\text { toda la planta a veces } \\
\text { arreglados en espigas } \\
\text { terminales o axilares }\end{array}$ & $\begin{array}{l}\text { glomérulos axilares } \\
\text { arreglados en espigas } \\
\text { terminales o axilares }\end{array}$ & $\begin{array}{l}\text { glomérulos axilares } \\
\text { arreglados en espigas } \\
\text { terminales o axilares }\end{array}$ & $\begin{array}{l}\text { glomérulos axilares } \\
\text { arreglados en espigas } \\
\text { terminales o axilares, } \\
\text { paniculiformes }\end{array}$ & $\begin{array}{l}\text { glomérulos axilares } \\
\text { arreglados en espigas } \\
\text { terminales o axilares }\end{array}$ \\
\hline Simetría de la flor & zigomorfa & zigomorfa & zigomorfa & zigomorfa & actinomorfa \\
\hline Tipo de pistilo & $\begin{array}{l}\text { estigmas insertos } \\
\text { directamente en el ápice } \\
\text { atenuado del ovario }\end{array}$ & $\begin{array}{l}\text { estigmas insertos direc- } \\
\text { tamente en el ápice } \\
\text { atenuado del ovario }\end{array}$ & $\begin{array}{l}\text { estigmas insertos direc- } \\
\text { tamente en el ápice } \\
\text { atenuado del ovario }\end{array}$ & $\begin{array}{l}\text { estigmas insertos direc- } \\
\text { tamente en el ápice } \\
\text { atenuado del ovario }\end{array}$ & $\begin{array}{l}\text { estigmas insertos en } \\
\text { una depresión rodeada } \\
\text { por un anillo }\end{array}$ \\
\hline Número de estambres & $2-3$ & $2(-5)$ & $2(-5)$ & 5 & 5 \\
\hline $\begin{array}{l}\text { Apéndices en los } \\
\text { segmentos del perianto }\end{array}$ & $\begin{array}{l}\text { con alargamientos } \\
\text { apicales como } \\
\text { cornículos y alados } \\
\text { transversalmente en } \\
\text { la base }\end{array}$ & $\begin{array}{l}\text { con alargamientos } \\
\text { apicales como } \\
\text { cornículos y alados } \\
\text { transversalmente en } \\
\text { la base }\end{array}$ & $\begin{array}{l}\text { con alargamientos } \\
\text { apicales como } \\
\text { cornículos y alados } \\
\text { transversalmente en } \\
\text { la base }\end{array}$ & $\begin{array}{l}\text { con quillas } \\
\text { longitudinales abaxiales } \\
\text { y alados } \\
\text { transversalmente en } \\
\text { la base }\end{array}$ & ausentes \\
\hline $\begin{array}{l}\text { Tamaño de la semilla } \\
(\mathrm{mm})\end{array}$ & $0.9-1.6$ & $0.8-1.7$ & $1-1.5$ & $0.7-1.1$ & $0.5-2$ \\
\hline Número cromosómico & $2 n=54$ & $2 n=36$ & ? & $?$ & $2 n=18$ \\
\hline
\end{tabular}


observaciones de campo que hemos realizado, tanto en la localidad tipo de S. mexicana en terrenos de la ex-Hacienda Angostura en Rio Verde, San Luis Potosí, poblaciones en Cuatrociénegas, Coahuila, así como de poblaciones bajo cultivo en el Colegio de Posgraduados, nos permiten determinar, sin duda alguna, que las plantas de $S$. mexicana son perennes como otras especies de $S$. sect. Brezia, tales como S. esteroa Ferren \& S.A.Whitmore y S. linearis (Elliott) Moq., de las que difiere por tener segmentos del perianto con ornamentaciones. Reed (1979) consideró a S. mexicana como una gipsófila halofítica distribuida del oeste de Texas a San Luis Potosí; nuestras colectas recientes de esta especie provienen de suelos salino-yesosos de Coahuila y San Luis Potosí.

Consecuentemente, las plantas anuales de Suaeda de Guanajuato, Jalisco, Michoacán, Estado de México, Distrito Federal, Tlaxcala y Puebla, no pueden ser identificadas como $S$. mexicana o como $S$. nigra. Estas plantas comparten características con S. calceoliformis (Hooker) Moq. y S. occidentalis (S.Watson) S.Watson, pero difieren por caracteres que son considerados para reconocer una especie nueva de Suaeda. Las características que distinguen a S. edulis de las especies con las que se ha confundido y de las especies con las que comparte caracteres se resumen en el Cuadro 1 con base en Ferren y Schenk (2004), Schütze et al. (2003) y observaciones personales. La presencia de $S$. calceoliformis en México fue reportada por primera vez por Ferren y Schenk (2004) sin precisar localidades, mientras que S. occidentalis no ha sido registrada para el país.

Ejemplares adicionales examinados: México. Guanajuato, Mpio. Valle de Santiago, Cráter-lago. Hoya del Rincón de Paranguero, $7 \mathrm{~km}$ al oeste de Valle, 1,850 m, cráter con afloramientos rocosos, ígneos y vegetación de bosque tropical caducifolio, playa del lado Sureste del cráter, 3/01/1989, $L$. I. Aguilera G. 169 (CHAPA). Jalisco, Mpio. Zacoalco de Torres, orilla de la Laguna de San Marcos, pastizal salino de Distichlis, 08/08/1986, Zamudio y Guevara 4207 (MEXU). Mpio. Atoyac, Laguna de Sayula, 30/10/2010, H. FloresOlvera y R. Noguez 1607 (CHAPA, ENCB, MEXU); 3 km al oeste de Atoyac en la base de la isla chica, $1,350 \mathrm{~m}$, llano salino con pastizal, 05/12/1993, M. Cházaro B. y R. Acevedo R. 722 (CHAPA). Michoacán, Mpio. Queréndaro, cerca de la estación del tren de Queréndaro, creciendo en suelo húmedo en la zona de halófitas, 26/07/1986, J. Rojas 86 (MEXU); Mpio. Copándaro, en el borde del lago en zona de halófitas, 27/07/1986, J. Rojas 95 (MEXU), Mpio. de Cuitzeo, La Palma, orilla sur del Lago de Cuitzeo, 1,950 m, junto a la carretera, pastizal halófilo en orilla de la laguna, 30/06/1986, S. Zamudio 4085 (CHAPA). Estado de México, Mpio. Coacalco: San Lorenzo Tetlixquiac, campos con maíz, avena, remolacha y alfafa, suelos salinos, 02/12/1977, F. Espinosa García 474 (MEXU); Mpio. Texcoco, Terrenos del Colegio de Postgraduados, terrenos ubicados hacia el lago de Texco- co, vegetación ruderal, 26/09/2000, H. Vibrans 6705 (CHAPA, MEXU); Teotihuacán, 25/06/1950, E. Matuda 19230 (MEXU); Zumpango, en campo húmedo, 08/09/1950, E. Matuda 19710 (MEXU). Distrito Federal, Iztapalapa, en ladera húmeda cerca de canal, 11/11/1951, E. Matuda 25643 (MEXU); Xochimilco: Chinampas y campos adyacentes, 14/ 10/1977, F. Espinosa García y J. Sarukhán 333 (MEXU). Puebla, Lago Totolcingo, $4 \mathrm{~km}$ E de El Carmen Tequexquitla, 2,350 m, partes húmedas con pastizal halófilo de Distichlis spicata, 3/10/2012, M.H. Flores-Olvera et al. 1668 (B, CHAPA, MEXU, MO, TEX, UAM-I, XAL, XOLO).

\section{Agradecimientos}

Los autores reconocen el apoyo de las siguientes personas para la realización de este artículo, a Pedro Mercado por su colaboración en la investigación sobre números cromosómicos de las especies de Suaeda en México, a José Luis Zárate Castrejón por el apoyo en trabajo de campo y observaciones, a Albino Luna por la ilustración, a Helga Ochoterena por elaborar las figuras, a Carlos Gómez Hinostrosa por elaborar el mapa, a Brenda Jéssica Zárate Castrejón por proporcionar la fotografía de Suaeda mexicana, a Fernando Chiang por la revisión del manuscrito y a Steven Koch por sus comentarios y su amabilidad durante la consulta del Herbario CHAPA. También agradecemos a los revisores anónimos que con sus acertados comentarios enriquecieron el manuscrito.

\section{Literatura citada}

Bye R. y Linares E. 2000. Los quelites, plantas comestibles de México: una reflexión sobre intercambio cultural. CONABIO. Biodiversitas 31:11-14.

Calderón de Rzedowski G. 1979. Chenopodiaceae. En: Rzedowski J. y Calderón de Rzedowski G. Eds. Flora Fanerogámica del Valle de México. Vol. I. CECSA, México, D.F.

Calderón de Rzedowski G. 2001. Chenopodiaceae. En: Calderón de Rzedowski G. y Rzedowski J. Eds. Flora Fanerogámica del Valle de México, pp. 115-124, 2ª . ed. Instituto de Ecología, A.C. y Comisión Nacional para el Conocimiento y Uso de la Biodiversidad, Pátzcuaro.

Espinosa F.J. y Sarukhán J. 1997. Manual de Malezas del Valle de México. Ediciones Científicas Universitarias UNAM-Fondo de Cultura Económica, México, D.F.

Ferren W.R. y Schenk H.J. 2004. Suaeda. En: Flora of North America Editorial Committee Ed. Flora of North America. North of Mexico. Vol. 4, pp. 390-398, Oxford University Press, Nueva York.

Hanan-Alipi A.M., Mondragón-Pichardo J. y Vibrans H. 2009. Malezas de México, Suaeda torreyana S.Watson. http://www. conabio.gob.mx/malezasdemexico/chenopodiaceae/suaeda-torreyana/fichas/ficha.htm

Hopkins C.O. y Blackwell W.H. 1977. Synopsis of Suaeda (Chenopodiaceae) in North America. Sida 7:147-173.

Lomonosova M. y Freitag H. 2003. A new species of Suaeda (Chenopodiaceae) from the Altai, Central Asia. Willdenowia 33:139-147. 
Martínez M. 1979. Catálogo de Nombres Vulgares y Científicos de Plantas Mexicanas. Fondo de Cultura Económica, México, D.F.

Martínez M. y Matuda E. 1979. Flora del Estado de México. Biblioteca Enciclopédica del Estado de México, México, D.F.

Pedrol J. y Castroviejo S. 1988. A propósito del tratamiento taxonómico y nomenclatural del género Suaeda Forsskal ex Scop. (Chenopodiaceae) en "Flora Iberica". Anales Jardín Botánico de Madrid 45:93-102.

Ramírez J. y Alcozer G.V. 1902. Sinonimia Vulgar y Científica de las Plantas Mexicanas. Oficina Tipográfica de la Secretaria de Fomento, México, D.F.

Reed C.F. 1979. Chenopodiaceae. En: Correll D.S. y Johnston M.C. Eds. Manual of the Vascular Plants of Texas, pp. 527-551, Texas Research Fundation, Austin.

Reiche C. 1977. Flora Excursoria en el Valle Central de México. $2^{a}$ ed. Manuel Porrúa, S.A., México, D.F.

Rzedowski J. 1978. La Vegetación de México. Limusa, México, D.F.

Rzedowski J. y Equihua M. 1987. Atlas Cultural de México: Flora. Secretaria de Educación Pública, Instituto Nacional de Antropología e Historia, Grupo Editorial Planeta, México, D.F.

Sánchez O. 1978. La Flora del Valle de México. Editorial Herrero, México, D.F.

Schütze P., Freitag H. y Weising K. 2003. An integrated molecular and morphological study of the subfamily Suaedoideae Ulbr. (Chenopodiaceae). Plant Systematics and Evolution 239:257286.

Standley P.C. 1916. Chenopodiaceae. En: Britton N.L., Murrill W.A. y Barnhart J.H. Eds. North American Flora 21 part 1, pp. 3-93, The New York Botanical Garden, Nueva York.

Recibido: 6 de junio de 2012

Aceptado: 10 de octubre de 2012 